\title{
Clinically Relevant Pharmacological Strategies That Reverse MDMA-Induced Brain Hyperthermia Potentiated by Social Interaction
}

\author{
Eugene A Kiyatkin ${ }^{*,}$, Suelynn Ren', Ken T Wakabayashi', Michael H Baumann' and Yavin Shaham' \\ 'National Institute on Drug Abuse - Intramural Research Program, NIH, Baltimore, MD, USA
}

\begin{abstract}
MDMA-induced hyperthermia is highly variable, unpredictable, and greatly potentiated by the social and environmental conditions of recreational drug use. Current strategies to treat pathological MDMA-induced hyperthermia in humans are palliative and marginally effective, and there are no specific pharmacological treatments to counteract this potentially life-threatening condition. Here, we tested the efficacy of mixed adrenoceptor blockers carvedilol and labetalol, and the atypical antipsychotic clozapine, in reversing MDMA-induced brain and body hyperthermia. We injected rats with a moderate non-toxic dose of MDMA $(9 \mathrm{mg} / \mathrm{kg})$ during social interaction, and we administered potential treatment drugs after the development of robust hyperthermia $\left(>2.5^{\circ} \mathrm{C}\right)$, thus mimicking the clinical situation of acute MDMA intoxication. Brain temperature was our primary focus, but we also simultaneously recorded temperatures from the deep temporal muscle and skin, allowing us to determine the basic physiological mechanisms of the treatment drug action. Carvedilol was modestly effective in attenuating MDMA-induced hyperthermia by moderately inhibiting skin vasoconstriction, and labetalol was ineffective. In contrast, clozapine induced a marked and immediate reversal of MDMA-induced hyperthermia via inhibition of brain metabolic activation and blockade of skin vasoconstriction. Our findings suggest that clozapine, and related centrally acting drugs, might be highly effective for reversing MDMA-induced brain and body hyperthermia in emergency clinical situations, with possible life-saving results. Neuropsychopharmacology (2016) 4I, 549-559; doi:I 0.1038/npp.20 I5. I 82; published online I5 July 2015
\end{abstract}

\section{INTRODUCTION}

Robust hyperthermia is the most dangerous symptom of acute MDMA intoxication, often leading to serious health complications and even death (Kalant, 2001; Gowing et al, 2002; Hall and Henry, 2006). The temperature effects of MDMA are relatively modest when the drug is administered to humans or animals under standard laboratory conditions (Mechan et al, 2002; Freedman et al, 2005; Liechti, 2014), but are strongly potentiated under conditions of high activity, social interaction, and warm ambient temperature (Gordon et al, 1991; Brown and Kiyatkin, 2004; Parrott, 2012). This state-dependent modulation of MDMA-induced hyperthermia renders MDMA much more dangerous when used during 'rave parties' or crowded events, both of which are associated with intense social interaction, increased physical activity, emotional engagement, and typically take place in warm, humid environments that diminish heat loss from body surfaces (Kalant, 2001; Parrott and Young, 2014).

We previously demonstrated that MDMA acts through two primary physiological mechanisms to induce

*Correspondence: Dr EA Kiyatkin, Behavioral Neuroscience, NIDA-IRP, 333 Cassell Drive, Baltimore, MD 21224, USA, Tel: + I 4107402844 , Fax: + 410740 2155, E-mail: ekiyatki@intra.nida.nih.gov

Received 16 April 2015; revised 2 June 2015; accepted 17 June 2015; accepted article preview online 24 June 2015 hyperthermia: metabolic brain activation, which increases intra-brain heat production, and skin vasoconstriction that prevents proper heat dissipation to the external environment (Brown and Kiyatkin, 2004; Kiyatkin et al, 2014). MDMAinduced hyperthermia can be life-threatening and current strategies for treatment, such as whole-body cooling, replenishment of water, and sedation with benzodiazepine tranquillizers, are merely palliative (Liechti, 2014). Therefore, there is a clear clinical need for pharmacological interventions to rapidly alleviate MDMA-induced hyperthermia in emergency room situations.

Here, we used MDMA at a moderate, non-toxic dose under conditions that mimic human drug use and examined the ability of three clinically approved medications to reverse MDMA-induced brain hyperthermia in awake, behaving rats. We assessed the effects of clozapine, an atypical neuroleptic, and carvedilol and labetalol, mixed alpha- and beta-adrenoceptor blockers. These medications are routinely used to treat chronic health problems in humans, and were chosen because of their preclinical success in attenuating MDMA-induced body hyperthermia (Blessing et al, 2003; Sprague et al, 2005). Clozapine acts on multiple neural receptors and glial cells in the brain, presumably inhibiting MDMA-induced metabolic activation, sympathetic tone, and centrally mediated vasoconstriction (Baldessarini and Frankenburg, 1991; Breier et al, 1994). Carvedilol and labetalol act peripherally to dilate skin vessels by 
blocking alpha- and beta-adrenoceptors (Sponer et al, 1992; Bakris, 2009).

Brain temperature was our primary focus, but we also simultaneously recorded temperatures from the temporal muscle and facial skin to determine the basic physiological mechanisms underlying brain temperature responses. This three-point recording technique allowed us to evaluate the effects of the drugs on intra-brain heat production due to metabolic neural activation and heat loss due to changes in peripheral vascular tone (Kiyatkin, 2010). Our findings demonstrate that drugs acting in the brain (ie, clozapine) are significantly more effective at blocking MDMA-induced peripheral vasoconstriction and reversing drug-induced brain hyperthermia than drugs acting directly on skin blood vessels (ie, carvedilol, labetalol). These preclinical data support the use of clozapine, or similar drugs, to reverse pathological brain and body hyperthermia caused by acute MDMA intoxication in humans, possibly with life-saving results.

\section{MATERIALS AND METHODS}

\section{Subjects, Surgery, and Thermocouple Sensors}

Long-Evans male rats ( 3-4 months old, $450 \pm 30 \mathrm{~g}, n=27$ ) were housed individually (12-h light cycle beginning at 07:00) with free access to food and water. All experiments complied with the 'Guide for the care and use of laboratory animals' (8th edition); protocols were approved by the NIDA-IRP Animal Care and Use Committee.

Rats underwent the three-point thermocouple electrode implantation procedure described in detail elsewhere (Kiyatkin et al, 2014). In brief, under general anesthesia, we implanted miniature copper-constantan thermocouple probes $(125 \mu \mathrm{m}$ in diameter) in the nucleus accumbens (NAc) shell $(\mathrm{AP}=1.2 \mathrm{~mm} ; \mathrm{L}=0.9 \mathrm{~mm} ; \mathrm{DV}=7.2-7.4 \mathrm{~mm})$, deep temporal muscle, and subcutaneously along the nasal ridge with the tip $\sim 15 \mathrm{~mm}$ anterior to bregma. We chose the NAc, a critical structure of the brain motivation-reinforcement circuit (Mogenson et al, 1980; Wise and Bozarth, 1987; Di Chiara, 2002), as a representative deep brain structure. We secured the probes with dental cement to three stainlesssteel screws threaded into the skull. Experiments began 5 days after surgery and were conducted during the light phase of the cycle (10:00-17:00). The cages were equipped with four infrared motion detectors (Med Associates) to measure locomotor activity. Temperature data were continuously recorded at a 10-s resolution using Thermes-16 (Physitemp Instruments). Rats were habituated for two or three 6-h sessions before and after surgery and for $\sim 1.5 \mathrm{~h}$ before the experimental manipulations.

\section{Experimental Protocol}

Our experimental protocol has three important features. First, we exposed rats to MDMA at a relatively modest, nontoxic dose (9 mg/kg or 1/5 of LD50; (Hardman et al, 1973)) and delivered the drug subcutaneously (in $0.3 \mathrm{ml}$ of saline), providing the slowest pharmacokinetics, analogous to oral consumption in humans (Baumann et al, 2009). Second, in contrast to most studies that utilized drug administration in quietly resting laboratory animals, we administered MDMA during social interaction with another rat at the height of 'psychophysiological activation,' when brain and body temperatures are significantly increased (Brown and Kiyatkin, 2004; Kiyatkin et al, 2014). This protocol is more relevant for human conditions because MDMA is recreationally used in social settings associated with high arousal (eg, rave parties, music festivals). Third, in contrast to most studies, where a treatment drug was administered before or at the same time as MDMA (Yeh, 1997; Sprague et al, 2005; Shioda et al, 2008; Hysek et al, 2012; Taffe, 2012), we injected each of the three test drugs-clozapine, carvedilol, and labetalol-after the MDMA injection, when brain and body temperatures were already significantly increased $\left(>38^{\circ} \mathrm{C}\right)$. This dosing regimen closely mimics the clinical situation, in which MDMA-intoxicated patients are treated for pathological hyperthermia in hospital emergency rooms.

Although we strived for a fully factorial, within-subjects design, the variability associated with MDMA temperature response made this difficult. However, generally we exposed all rats to a 1-week-long experimental protocol where they randomly received MDMA-saline (control), MDMAtreatment drug, saline, or treatment drug alone on alternating days. In rats from the first two groups, we injected MDMA 10 min after the onset of the 1-h social interaction followed by a counterbalanced injection of either a treatment drug (clozapine, carvedilol, and labetalol) or saline. In rats from two other groups, we injected either a treatment drug (clozapine, carvedilol, and labetalol) or saline under quiet resting conditions. Each rat received only two MDMA injections, either alone or with a treatment drug. Each morning, we took rats from their home cages, placed them in recording chambers, and connected them to the Thermes-16 recording apparatus. Following at least $1.5 \mathrm{~h}$ of habituation (when temperatures typically normalized at their 'basal levels'), we placed a novel, drug-naive conspecific male in the experimental chamber, which denoted the start of a 60-min 'social interaction' session. We injected MDMA 10 min after the start of social interaction, which coincides with the maximal temperature response during this procedure (Kiyatkin, 2010). The two rats freely interact and engage in social behaviors such as sniffing, rearing, and orogenital contacts. Approximately $50 \mathrm{~min}$ after MDMA injection (end of social interaction), we removed the drug-naive rat. Given the variability of the MDMA-induced temperature response, the moment of either saline or treatment drug injection was determined by an acceleration of brain temperature reaching $\sim 38.5^{\circ} \mathrm{C}$, the upper limits of physiological increases (Kiyatkin, 2005). We dissolved clozapine in saline and adjusted the $\mathrm{pH}$ to 5.5-5.7 with $\mathrm{HCl}$. We also dissolved labetalol in saline and carvedilol in a $3: 1$ volume ratio of DMSO:PBS. We injected all 'treatment' drugs intraperitoneally at the same dose $(5 \mathrm{mg} / \mathrm{kg}, 1 \mathrm{ml} / \mathrm{kg}$ volume); this dose was chosen based on previous studies (Blessing et al, 2003; Sprague et al, 2005).

We also examined the effects of each treatment drug alone under quiet resting conditions. Following $\sim 1.5 \mathrm{~h}$ of habituation in the testing cage, we injected the rat with clozapine, carvedilol, labetalol, or saline, at the same doses and volumes as described above.

\section{Data Analyses}

We analyzed temperature changes in terms of absolute values in each recording location (ie, NAc, temporal muscle, skin), 
relative temperature changes with respect to baseline, as well as NAc muscle and skin muscle temperature differentials. As the brain and temporal muscle receive arterial blood from the same common carotid artery and are equally exposed to blood-delivered heat from the body, NAc minus muscle temperatures (NAc-muscle differentials) show the source of heat production, providing a measure of drug-induced metabolic brain activation. Skin temperature is not only determined by the state of peripheral vessels, but it also depends on the temperature of arterial blood inflow. Therefore, skin minus muscle temperatures (skin-muscle differentials) serve as an accurate measure of peripheral vascular tone, another important determinant of brain temperature changes (Kiyatkin, 2010). We used one-way ANOVA with repeated measures, followed by Fisher's PLSD post hoc test to evaluate the effects of treatment drugs on the different dependent measures. We used two-way repeated measures ANOVA to examine the differences in the effects of each treatment drug $v s$ saline. Finally, we also evaluated the efficacy of treatment by determining the difference in the effects of each treatment drug with respect to the control group (MDMA-saline). This comparison allowed us to assess the onset, strength, and duration of a presumed therapeutic effect.

\section{RESULTS}

\section{Effects of MDMA During Social Interaction on Brain and Body Temperature Homeostasis}

Humans typically use MDMA under conditions of high psychosocial and physiological activation. To model this human condition, we examined the effects of MDMA at a moderate dose $(9 \mathrm{mg} / \mathrm{kg}, \mathrm{sc})$ during social interaction with another rat, and injected saline when brain temperature exceeded its physiological range $\left(\sim 38.5^{\circ} \mathrm{C}\right)$. We used these 'control' data to assess the effects of each 'treatment drug'.

Consistent with our previous studies (Brown and Kiyatkin, 2004; Kiyatkin et al, 2014), MDMA injected during social interaction induced highly variable increases in NAc temperature (Figure 1a) that were independent of both body weight $(r=0.30, P=0.23)$ and basal temperatures $(r=0.23$, $P=0.37)$. From the 18 rats of this group, 4 rats showed lethal increases in brain temperature $\left(40.5-42.2^{\circ} \mathrm{C}\right)$, dying either within the 6-h post-injection recording session or overnight (Figure 1b). For the surviving rats $(n=14)$, mean NAc temperature rapidly increased during social interaction (time $=0 \mathrm{~min}$ ) and was significantly higher $v s$ baseline $\left(37.87 \pm 0.11^{\circ} \mathrm{C}\right.$ vs $36.80 \pm 0.19 ; P<0.01$ or $1.36 \pm 0.22{ }^{\circ} \mathrm{C}$ above baseline) at the time of MDMA injection (time $=$ $10 \mathrm{~min}$ ) (Figure 2a and b). After MDMA injection, NAc temperature initially decreased, but began to gradually increase again, reaching its peak $\left(39.05 \pm 0.31{ }^{\circ} \mathrm{C}\right.$, or $2.54 \pm$ 0.41 above baseline) at $\sim 130 \mathrm{~min}$ post injection. Subsequently, NAc temperature slowly decreased, but remained significantly higher than the initial baseline $\left(37.35 \pm 0.31^{\circ} \mathrm{C}\right.$ vs $36.80 \pm 0.19 ; P<0.01)$ at the end of recording session (300 min). Temperature dynamics in the temporal muscle and skin were similar to those in the NAc such that two peaks, corresponding to the social interaction procedure and MDMA-induced hyperthermia, were clearly visible in each temperature measure. Temperature increases were greatest
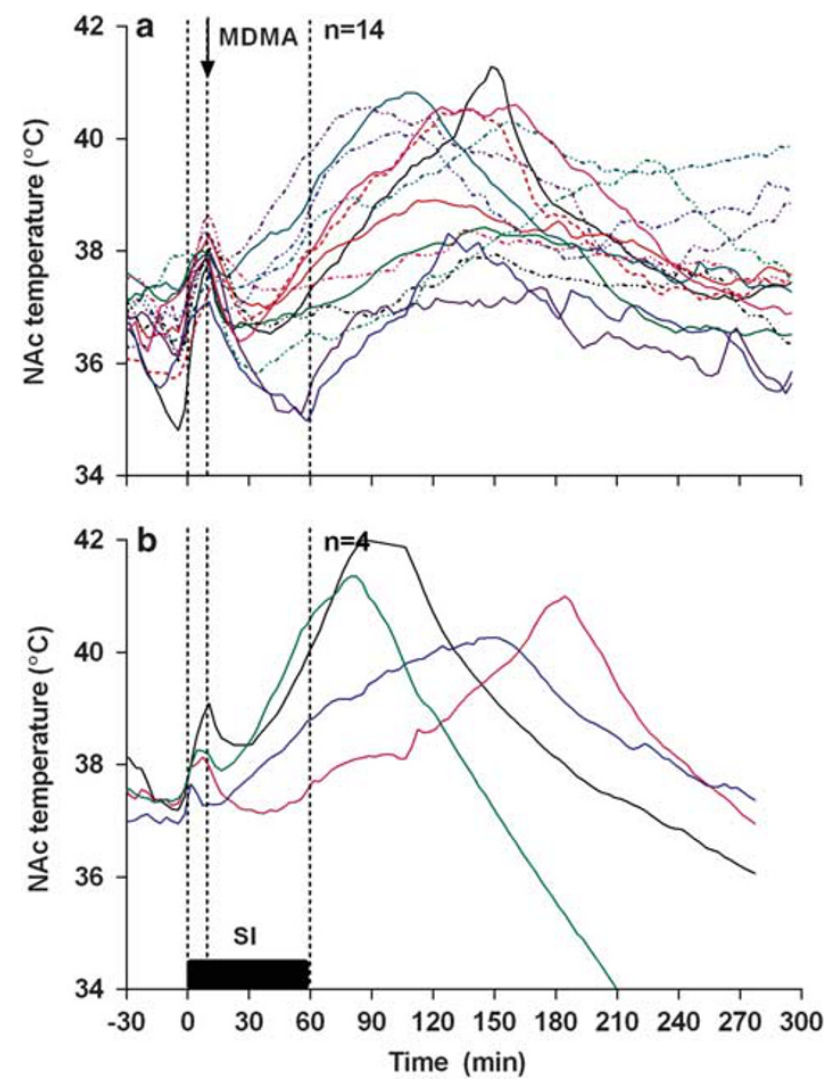

Figure I Variability of brain hyperthermic effects induced by MDMA administered during social interaction. Top panel (a): changes in nucleus accumbens (NAc) temperature in each of the 14 rats that survived after administration of MDMA; bottom panel (b): changes in NAc temperature in the four rats that died after MDMA exposure. Two vertical hatched lines show onset and offset of 60-min social interaction and a middle hatched line with arrow shows the time of MDMA administration. SI: social interaction.

in the NAc, weaker in the temporal muscle, and weakest in the skin (Figure 2b).

Although brain and muscle temperatures transiently decreased after MDMA injection, this reduction is similar to the effect of saline injection under the same conditions. Thus, the initial decrease is not a pharmacological effect of MDMA, but is related to social interaction dynamics and injection procedure. We have shown previously that the effect of MDMA on temperature parameters is in fact monophasic subtracting values obtained from rats injected with saline during social interaction (Kiyatkin et al, 2014).

NAc-muscle temperature differential, a measure of metabolic brain activation, rapidly increased as a result of social interaction and later stabilized post MDMA injection at values $\sim 0.4^{\circ} \mathrm{C}\left(\mathrm{F}_{13,1413}=4.3, P<0.01\right.$; Figure $\left.2 \mathrm{c}\right)$. Skinmuscle temperature differential, a measure of skin vasomotor tone, phasically decreased during social interaction and showed a second decrease after the MDMA injection, dropping to its nadir $\left(\sim 1.2^{\circ} \mathrm{C}\right)$ at the time of maximal brain and muscle temperature increase $\left(\mathrm{F}_{13,1413}=5.8, P<0.01\right.$; Figure 2c). Subsequently, the skin-muscle differential slowly returned toward baseline, but remained significantly different from basal values at the end of the session.

MDMA injections during social interaction induced relatively weak and highly variable increases in locomotor 

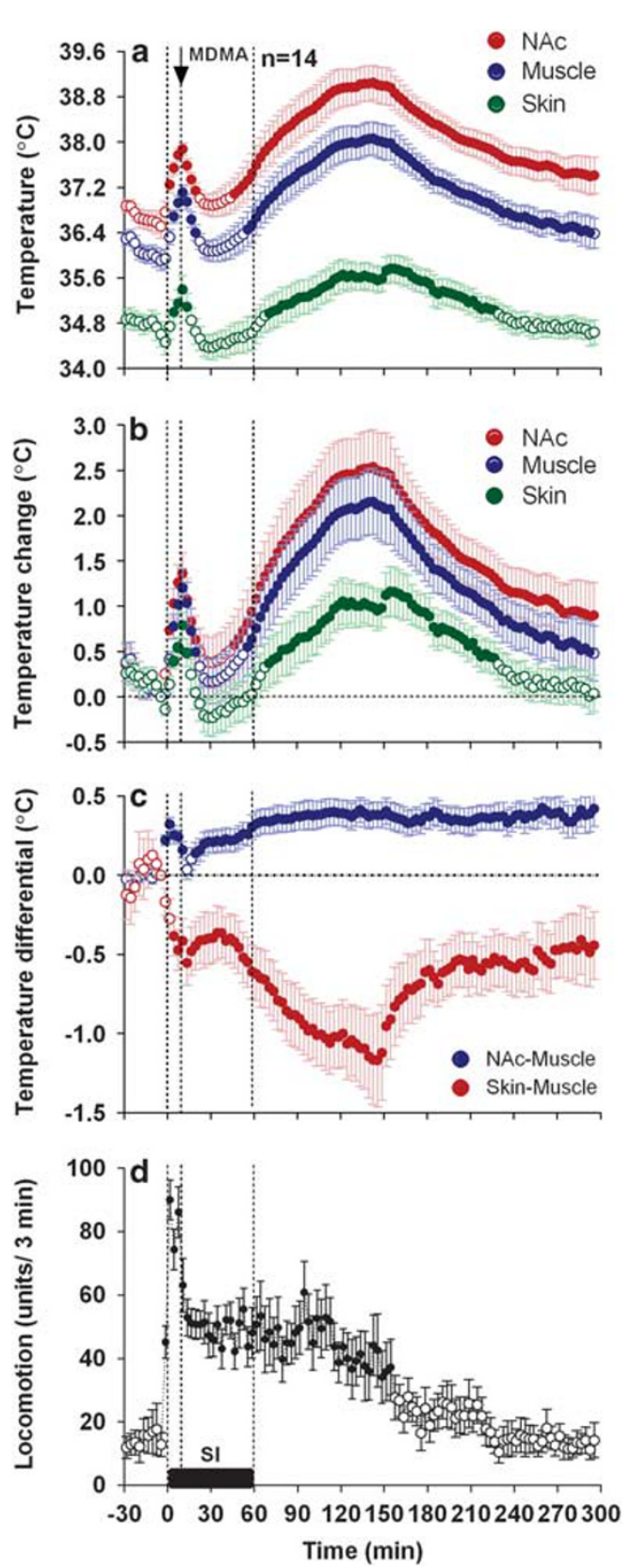

Figure 2 Mean temperature responses induced by MDMA administered during social interaction. (a) Mean changes in temperatures recorded from the NAc, temporal muscle, and skin; (b) Mean changes relative to the initial, pre-social interaction baseline $\left(=0^{\circ} \mathrm{C}\right)$; (c) Mean changes in NAc-muscle and skin-muscle temperature differentials; (d) Mean changes in locomotion. First and last vertical hatched lines show the onset and offset of social interaction, and a middle hatched line with arrow shows the time of MDMA injection. The effects of social interaction+MDMA on all parameters evaluated using one-way repeated measure ANOVA (effect of time) were highly significant $(P$-values $<0.0$ I); individual significant points (Fisher's PLSD test) are shown as filled symbols; $n$ is number of rats in each group. SI: social interaction.

activity for $\sim 140 \mathrm{~min}$ post injection (Figure $2 \mathrm{~d}$ ). The rats' activity rapidly increased at the start of social interaction and gradually decreased to a moderate level that was maintained post MDMA injection. The effect of MDMA on locomotion is monophasic: the initial surge in locomotor activity and subsequent decrease is an expected effect of social interaction, as is evident when evaluating locomotor activity changes after saline+social interaction activity values are subtracted from MDMA+social interaction activity values (Kiyatkin et al, 2014).

\section{Effects of Clozapine, Carvedilol, and Labetalol on MDMA-Induced Hyperthermia}

Next, we examined the effects of clozapine, carvedilol, and labetalol on MDMA-induced hyperthermia. In each case, the treatment drug was injected at variable times after MDMA injection (mean, $83 \mathrm{~min}$, range, 57-140 $\mathrm{min}$ ), when brain temperature increased to near-pathological levels $\left(\sim 38.5^{\circ} \mathrm{C}\right)$. Owing to between-subject variability in responsiveness to MDMA, in some cases test drugs were injected at somewhat lower or higher temperatures. To assess the effects of treatment, data shown in Figures 3, 4 and 5 are normalized to the moment of treatment drug/saline injection (time $=0$ ). Temperature changes preceding time 0 represent the combined effects of MDMA+social interaction, and changes following time 0 represent treatment drug effects.

\section{Clozapine}

After clozapine injection, NAc temperature rapidly decreased (Figure 3e), resulting in a large difference $v s$ control (saline) (Figure 4a; two-way ANOVA: effects of time $\left(\mathrm{F}_{61,1403}=33.8\right.$, $P<0.01)$, treatment $\left(\mathrm{F}_{1,23}=55.3, P<0.01\right)$, and time $\times$ treatment interaction $\left.\left(\mathrm{F}_{61,1403}=14.2, P<0.01\right)\right)$. The clozapineinduced temperature decrease was rapid and profound; the final temperature values in the clozapine treatment group were lower $\left(35.83 \pm 0.36^{\circ} \mathrm{C}\right)$ than the initial baseline (Figure 3f) and significantly lower than in the control group that received MDMA with saline $\left(37.35 \pm 0.31^{\circ} \mathrm{C}, P<0.05\right)$. These values, however, remain within the physiological range; similarly low or even lower values occur in wellhabituated rats during day-time recording (Kiyatkin, 2010). Between-group differences appeared at the second data point (3-6 min), became significant from the fourth data point ( $\sim 10 \mathrm{~min})$, and were maintained at high levels during the entire post-injection interval (Figure 4a). Muscle and skin temperatures also decreased after clozapine injection (Figure $3 \mathrm{a}, \mathrm{b}$, e and $\mathrm{f}$ ), and the difference $v s$ control was also significant.

In contrast to the stable increase in NAc-muscle differentials present in the control group (Figure 3c), this parameter decreased after clozapine injection (Figure $3 \mathrm{~g}$ ). The decrease developed with $\sim 10-15 \mathrm{~min}$ onset latency, producing a significant difference $v s$ control from $\sim 30 \mathrm{~min}$ post injection (Figure 4b). However, the most rapid and strong effects of clozapine were found for the skin-muscle differentials, which reflect the vasomotor tone of skin vessels. Although this parameter further decreased after saline injection (Figure 3c), suggesting MDMA-induced sustained skin vasoconstriction, the skin-muscle differential began to rapidly increase immediately after clozapine injection (Figure 3g), reflecting a full blockade of drug-induced vasoconstriction. The difference between clozapine and saline appeared from the second data point (3-6 min) and this difference continued to increase throughout the entire post-treatment interval. Finally, clozapine inhibited 

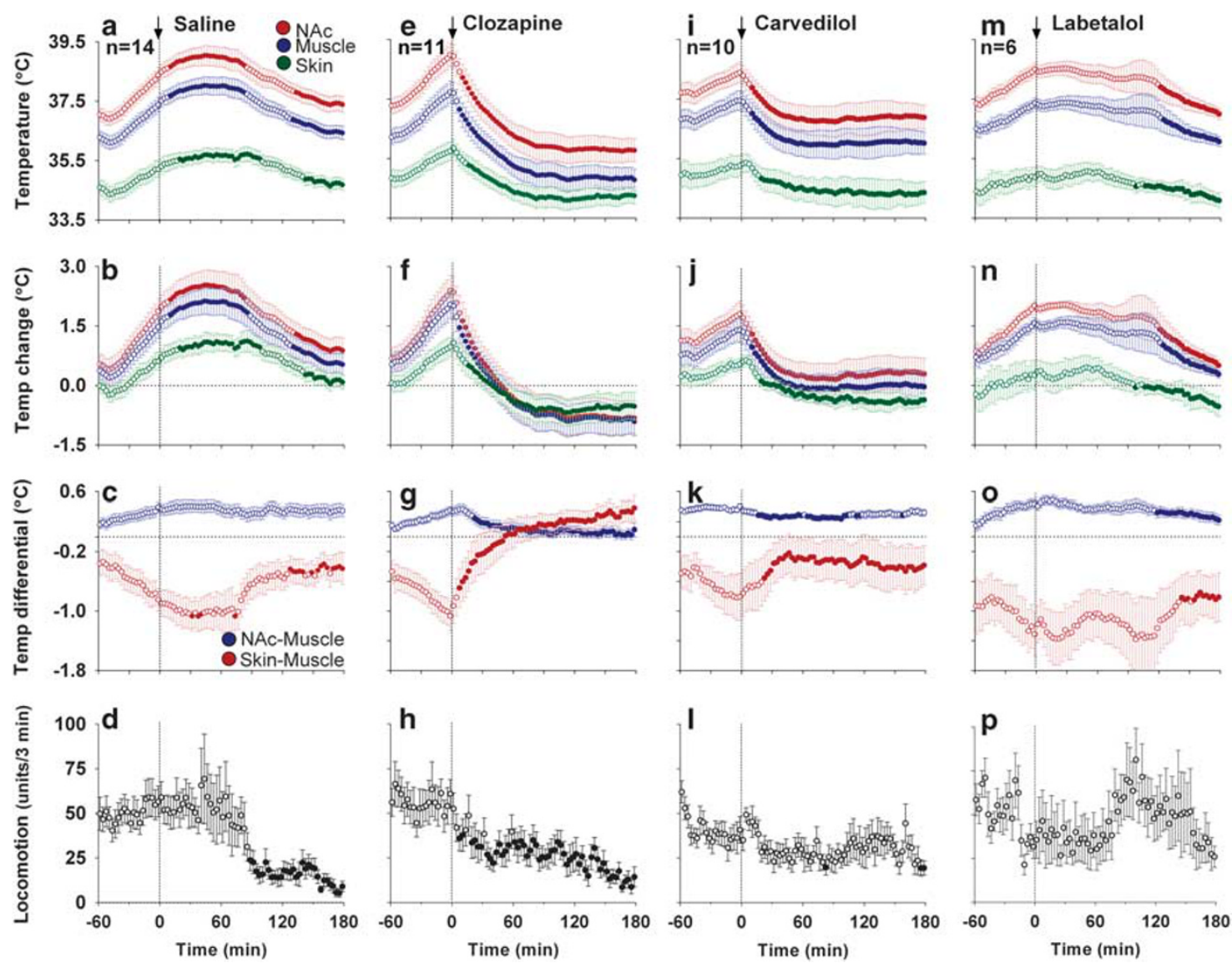

Figure 3 The effects of clozapine, carvedilol, and labetalol on MDMA-induced temperature and locomotor responses. (a-d) MDMA-saline: absolute temperatures, relative temperatures, NAc-muscle and skin-muscle differentials, and locomotion. (e-h) MDMA-clozapine, (i-l) MDMA-carvedilol, and (m- $p$ ) MDMA-labetalol. The graphs show changes in different parameters before and after administration of each testing drug and saline ( 0 min). In each group, the rats received a single injection of MDMA and the testing drug/saline was injected at the time of gradual NAc temperature increase in the range of $38.5^{\circ} \mathrm{C}$ (mean $83 \mathrm{~min}$, range 57-140 min). Filled symbols show values significantly different from the last pre-treatment value; the absence of filled symbols indicates the absence of a significant effect on a specified parameter evaluated with one-way ANOVA, n: number of rats.

MDMA-induced hyperlocomotion (Figure 3d) while maintaining a normal level of locomotion similar to that seen before MDMA injections (see Figure 2d). In contrast to the saline treatment, where 4 out of 16 rats died after MDMA exposure, all 11 rats that received MDMA plus clozapine during social interaction were in good health in the days following an experiment.

\section{Carvedilol and Labetalol}

Carvedilol attenuated MDMA-induced increases in NAc, temporal muscle, and skin temperatures, returning these parameters to near-baseline levels by the end of the session (Figures 3i-1 and 4d). Carvedilol also attenuated the vasoconstrictive effects of MDMA as evidenced by a moderate increase $\left(\sim 0.7^{\circ} \mathrm{C}\right)$ in skin-muscle differentials, but this effect was short-lived and lasted only $\sim 100 \mathrm{~min}$ post treatment (Figure $4 \mathrm{f}$ ). Carvedilol minimally affected NAcmuscle differential (Figure 3k) and the effect of treatment $v s$ control was not significant (effect of time $\mathrm{F}_{61,1342}=1.2$, $P=0.16$; effect of treatment: $\mathrm{F}_{1,22}=2.2, \quad P=0.15$; see Figure 4e). Finally, carvedilol inhibited MDMA-induced locomotor activation, although locomotor activity was maintained at normal levels throughout the recording session (Figure 31). All 10 rats that received carvedilol did not show any health abnormalities during the session and in the following days.

Labetalol had no evident effects on any of the temperature responses caused by MDMA plus social interaction (Figure $3 \mathrm{~m}-\mathrm{p}$ ).

\section{Comparisons Across Drugs}

To further compare the efficacy of each drug in attenuating MDMA-induced hyperthermia, we calculated the mean differences between the effects of MDMA+treatment drug and MDMA+vehicle. In addition, we approximated temperature change, as analyzed by the integral of the difference between saline and drug treatment groups (ie, area under the curve) for the time of maximal effect ( $80 \mathrm{~min}$; Figure 5, right panel).

The attenuating effect of clozapine on NAc and muscle temperatures appeared with the shortest latencies $(\sim 6 \mathrm{~min})$, and displayed the greatest magnitude and longest duration (Figures $4 \mathrm{a}$ and $5 \mathrm{a}, \mathrm{b}$ ). Accordingly, the effect of clozapine on NAc and muscle temperatures was significantly stronger $(P<0.05)$ than the effects of carvedilol and labetalol (Figure $5 \mathrm{e}$ and $\mathrm{f}$ ). 

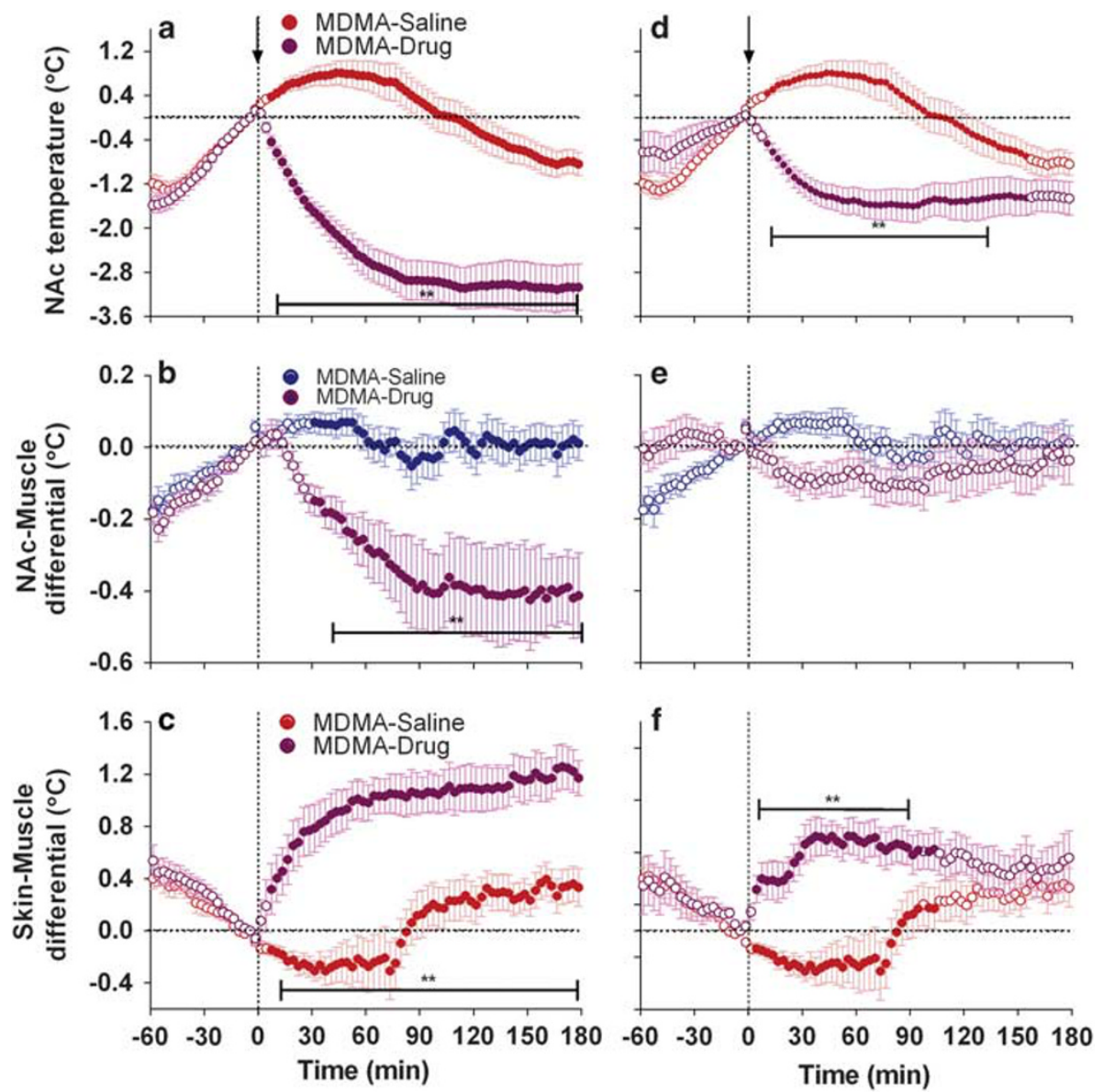

Figure 4 Between-condition differences in the effects of clozapine and carvedilol vs control treatment (saline). (a-c) MDMA-clozapine: relative NAc temperatures, NAc-muscle differentials, skin-muscle differentials; ( $d-f)$ MDMA-carvedilol. Each graph shows the change in the parameter after injection of the drug and saline. The between-condition differences were assessed by using two-way ANOVA with repeated measures; significant between-group differences are indicated by horizontal lines with asterisks and filled symbols in both groups.

Clozapine also has the greatest attenuating effects on MDMA-induced intra-brain heat production and skin vasoconstriction, showing the largest decrease in NAcmuscle differential and a strong, sustained increase in skinmuscle differential, respectively (Figure $5 \mathrm{c}, \mathrm{d}$, g and $\mathrm{h}$ ). In contrast, carvedilol had a much weaker effect on NAc-muscle differentials and a less pronounced, short-lived effect on skin-muscle differentials. Finally, labetalol had minimal effects on MDMA-induced changes in all temperature measures (Figure 5).

\section{The Effects of Clozapine or Carvedilol Alone on Brain and Body Temperature Homeostasis}

We examined the effects of clozapine or carvedilol on brain, muscle and skin temperatures, and locomotion when the drugs were injected under quiet resting conditions in the absence of social interaction and MDMA administration (Figure 6). Both drugs moderately decreased NAc and muscle temperatures and the effects of carvedilol were relatively stronger $\left(\sim 1.5^{\circ} \mathrm{C}\right)$ than the effects of clozapine $\left(\sim 1.0^{\circ} \mathrm{C}\right)$. The hypothermic effects were evident within $\sim 120$ min post injection, but carvedilol-induced temperature decreases were somewhat more prolonged (Figure $6 \mathrm{~b}$ ). In contrast to MDMA, which induced robust increases in NAc- muscle differentials, this parameter remained unchanged (close to zero) after injections of either clozapine or carvedilol (Figure 6c). However, skin-muscle differentials in both cases significantly increased (clozapine: $F_{10,1077}=2.1$; carvedilol: $\mathrm{F}_{5,581}=2.7, P<0.01$ ), indicating skin vasodilation. This effect was maintained throughout the entire postinjection period and was larger in magnitude for carvedilol than for clozapine. In contrast to the saline control, in which locomotion was relatively stable except for a transient, injection-induced rise, locomotor activity slightly decreased after carvedilol and clozapine injections; the inhibitory effect of clozapine was stronger than that of carvedilol $\left(\mathrm{F}_{5,581}=1.3\right.$, $P<0.05$ and $F_{10,1077}=1.8, P<0.01$ ) (Figure $6 \mathrm{~d}$ ).

\section{DISCUSSION}

Pathological hyperthermia is one of the most dangerous symptoms of acute MDMA intoxication and can cause serious health complications, including severe neurological impairment, rhabdomyolysis, organ failure, and even lethality (Kalant, 2001; Gowing et al, 2002; Hall and Henry, 2006). Although relatively rare, this response is unpredictable and strongly potentiated under common environmental and social conditions of recreational MDMA use. As the available 



Figure 5 Comparative effectiveness of different drugs in reversing MDMA-induced temperature responses. Left panel shows the time-course of the effects of each drug (difference vs saline) on (a) brain and (b) muscle temperatures as well as (c) NAc-muscle and (d) skin-muscle temperature differentials. Right panel shows the mean effects (area under curve for 80 min post injection) for each testing drug (e-h). Time-course data were analyzed using one-way ANOVA with repeated measures; filled symbols show the values significantly different from the last pre-treatment value (Fisher's PLSD test). Bar graph data were analyzed using one-way ANOVA; significant between-drug differences are shown by an asterisk $(* P<0.05 ; * * P<0.0$ I).

clinical strategies to counteract this life-threatening symptom are palliative and limited, there is an urgent need for a pharmacotherapy that could be used in acute, crisis situations to save the lives of highly intoxicated individuals. Our study was designed to test the efficacy of two pharmacological strategies for reversing brain and body hyperthermia induced by MDMA under conditions that mimic recreational drug use in humans. In a clinically relevant rat model of acute MDMA intoxication, we demonstrate the superior effectiveness of the antipsychotic medication clozapine in reversing
MDMA-induced brain and body hyperthermia, as compared with the peripherally acting alpha-beta adrenoceptor blockers carvedilol and labetalol.

Most previous studies with MDMA assessed body temperatures by using either rectal probes or intraperitoneal sensor implants. In contrast, we focused on brain temperature because the brain is the most temperature-sensitive organ (Rumana et al, 1998; Dewhirst et al, 2003) and the most harmful effects of MDMA are related to changes in brain activity and neural functions (Kiyatkin, 2010). In most 

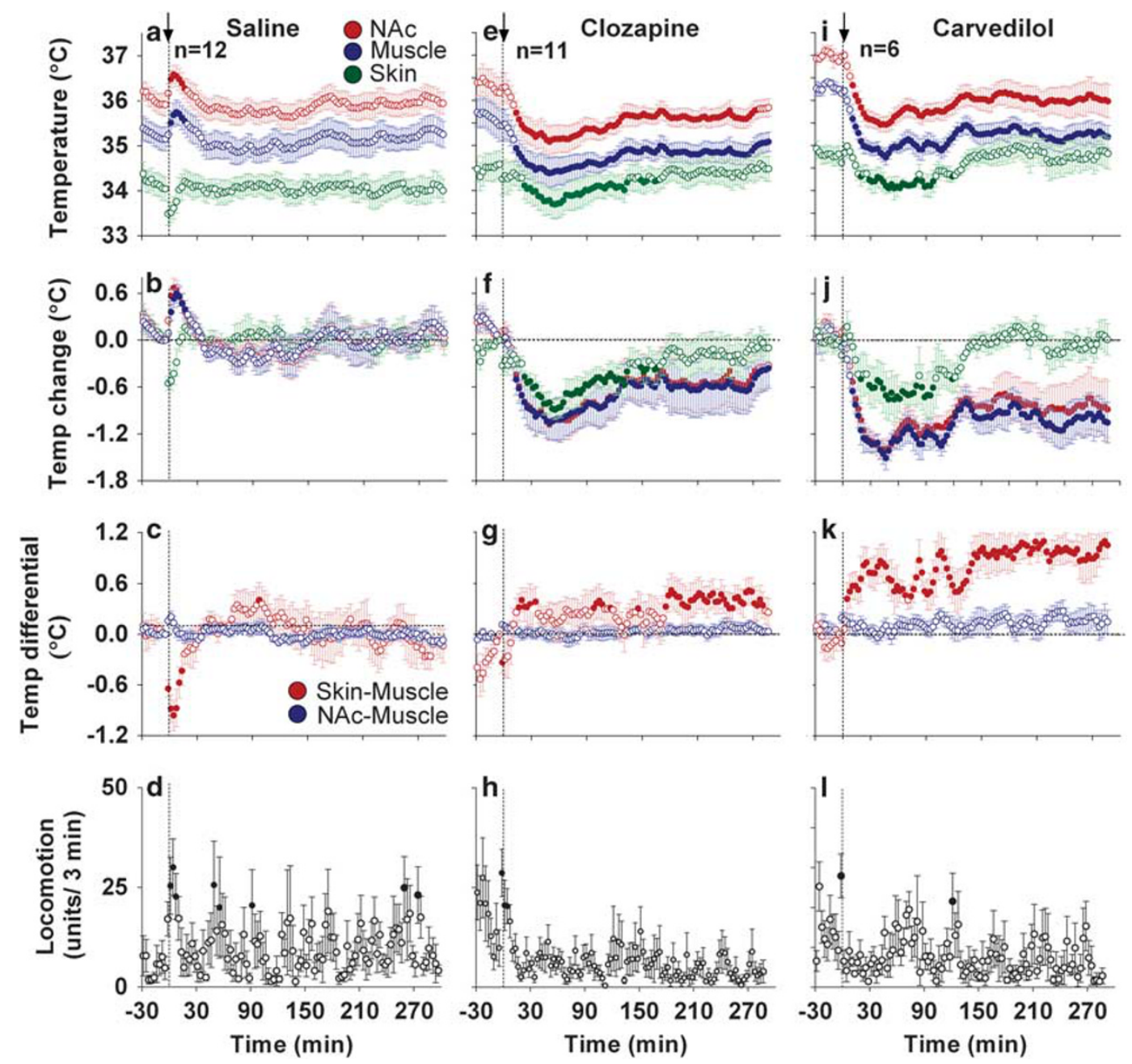

Figure 6 Mean temperature responses induced by clozapine $(5 \mathrm{mg} / \mathrm{kg})$ and carvedilol $(5 \mathrm{mg} / \mathrm{kg})$ administered to rats under quiet resting conditions. (a-d) Saline: absolute temperature changes, relative temperature changes, NAc-muscle and skin-muscle differentials, locomotion; (e-h) clozapine; and $(i-l)$ carvedilol. Saline injections induced a transient temperature responses associated with the injection procedure itself. Clozapine and carvedilol induced modest hypothermic and hypoactivity effects and significant increases in skin-muscle differentials ( $P$-values $<0.0 \mathrm{I}$ ), indicating peripheral vasodilation. Both drugs had no significant effects on NAc-muscle differentials and locomotion. $n$ is number of rats in each group.

previous studies, MDMA was used in high or multiple doses, but we administered MDMA subcutaneously at a moderate, non-toxic dose $(9 \mathrm{mg} / \mathrm{kg}$ or $1 / 5$ of the LD50) to mimic the pharmacokinetics of tablets taken by humans. Although this dose exceeds the typical range of recreational consumption (1-2 mg/kg; Parrott, 2005), because of the large onset latency of psychoactive effects, MDMA is sometimes taken by humans at higher doses or repeatedly with the total dose close to that used in our study. To model the social conditions of human drug use, we injected MDMA during social interaction, a procedure known to increase intra-brain heat production and induce peripheral vasoconstriction (Kiyatkin, 2010).

Instead of prophylactically pretreating the laboratory animals, we injected the treatment drugs when NAc temperatures began steadily increasing and reached near-pathological levels $\left(\sim 38.5^{\circ} \mathrm{C}\right)$, modeling a human-relevant, crisis-like situation. This treatment protocol allowed us to compare the effectiveness of each drug in terms of the onset of its therapeutic action, strength, and duration. All drugs tested in this study are FDA-approved, currently used to treat chronic diseases in humans, and have previously been shown to decrease MDMA-induced body hyperthermia under standard laboratory conditions (Blessing et al, 2003; Sprague et al, 2005; Hysek et al, 2012; Liechti, 2014).

\section{Targets and Pathways to Alleviate MDMA-Induced Hyperthermia}

The downstream pharmacological and biochemical mechanisms underlying MDMA-induced hyperthermia are complex and involve multiple factors, including excessive sympathetic activation, dopamine hyperactivity, decoupling of mitochondrial ATP, and heat production in both the brain and periphery (see Dao et al, 2014 for review). As such, rather than focusing on the specific downstream neurotransmitter, receptor, or signaling mechanisms, we instead chose to focus on the basic physiological mechanisms underlying MDMA-induced hyperthermia and its reversal by the treatment drugs. 
We confirmed that MDMA-induced brain hyperthermia results from two principle physiological mechanisms: increased intra-brain heat production due to metabolic neural activation and a centrally mediated peripheral vasoconstriction that prevents heat dissipation from the body surfaces (Kiyatkin et al, 2014). These two mechanisms are also transiently engaged under physiological conditions such as social interaction, resulting in phasic, relatively modest increases in brain and body temperatures $\left(\sim 1^{\circ} \mathrm{C}\right.$; Kiyatkin, 2010). MDMA administered during social interaction induces much stronger, tonic increases in intra-brain heat production coupled with exceptionally strong, sustained vasoconstriction, resulting in pathological hyperthermia. In this study, more than half of rats (10/18) showed brain temperature increases of $>40{ }^{\circ} \mathrm{C}$ and four of them died.

\section{Central Focus: Clozapine}

Clozapine $(1-5 \mathrm{mg} / \mathrm{kg})$ has been previously shown to decrease high-dose MDMA-induced body hyperthermia via vasodilation, as assessed by an ear pinna artery Doppler signal in rabbits (Blessing et al, 2003) and blood flow Doppler signal in the tails of rats (Blessing et al, 2003). Consistent with these data, we found that a low intraperitoneal dose of clozapine $(5 \mathrm{mg} / \mathrm{kg}$ or $\sim 2 \%$ of LD50; Lindt et al, 1971) completely reversed MDMA-induced brain and body hyperthermia. The temperature decrease was exceptionally rapid, appearing within $\sim 6 \mathrm{~min}$, and both brain and muscle temperatures returned to the initial, quiet resting baseline within $\sim 30 \mathrm{~min}$ post injection. Consistent with its central, antipsychotic action, clozapine strongly reduced MDMA-induced increases in NAcmuscle temperature differentials, suggesting a gradual decrease in intra-brain heat production due to blockade of druginduced metabolic brain activation. Within the first $3 \mathrm{~min}$ post treatment, clozapine also reversed the decreases in skin-muscle temperature differentials, suggesting a rapid blockade of skin vasoconstriction. In addition, clozapine decreased MDMAinduced locomotor activation while maintaining normal activity levels and showing no evident signs of sedation.

Clozapine administered alone under quiet resting conditions showed weak hypothermic effects coupled with a mild hypoactivity; both effects could be explained by the absence of preceding brain activation and a decreased activity state that naturally occurs in well-habituated, drug-free rats during day-time recordings (Kiyatkin, 2010). Consistent with this explanation, clozapine alone had no effects on NAc-muscle differentials but weakly increased skin-muscle differentials, reflecting a quiet-state skin vasodilation.

\section{Peripheral Focus: Carvedilol and Labetalol}

Sustained skin vasoconstriction appears to be an important mechanism underlying MDMA-induced brain and body hyperthermia. Therefore, we also tested the peripherally acting vasodilator drugs, carvedilol and labetalol. These mixed alpha-beta adrenoceptor blockers have similar pharmacodynamics and they directly dilate blood vessels (Sponer et al, 1992; Bakris, 2009), thus increasing heat dissipation from skin surfaces (Kellogg, 2006; Charkoudian, $2010)$. At a relatively low dose $(5 \mathrm{mg} / \mathrm{kg}$ or $0.6 \%$ of LD50; Carvedilol, 2012) under drug-free conditions, carvedilol moderately increased skin-muscle differentials, confirming skin vasodilation. Under these basal conditions, carvedilol also decreased NAc and muscle temperatures and this effect was stronger and more prolonged than that of clozapine. However, these temperature decreases remained within the physiological range seen in well-habituated, quietly resting rats during day-time recordings (Kiyatkin, 2010). Consistent with its peripheral action, carvedilol had no effect on either NAc-muscle differentials or locomotion.

Carvedilol also reversed MDMA-induced brain and body hyperthermia but its effects were slower, weaker, and more transient than those of clozapine. In contrast to the strong effects of clozapine on MDMA-induced increases in NAcmuscle differentials, peripherally acting carvedilol had virtually no effects on this metabolism-related parameter. Surprisingly, the effects of carvedilol on MDMA-induced changes in skin-muscle differentials, an index of cutaneous vascular tone, were weaker and incomplete compared with the effects of clozapine. Carvedilol also had minimal effects on MDMA-induced hyperlocomotion, and the rats' activity remained at normal levels, suggesting lack of sedative effects.

Human reports suggest that labetalol taken before MDMA exposure decrease MDMA-induced hyperthermia (Liechti, 2014), but in our hands this drug was ineffective and had minimal, if any, effects on all temperature parameters. The reasons for the differences between carvedilol and labetalol are unclear and may be due to their different affinities for the various subgroups of alpha- and beta-adrenoceptors (Ruffolo et al, 1990). As a vasodilator, labetalol also appears to be less potent than carvedilol (Tomlinson et al, 1987); therefore, the different effects of the drugs in our study may be because of the choice of a uniform drug dose. However, our exploratory tests with a higher dose of labetalol $(20 \mathrm{mg} / \mathrm{kg})$ did not reveal clear effects in attenuating MDMA-induced hyperthermia and also led to adverse side-effects (unpublished observation).

\section{Conclusions and Translational Relevance}

We show that a moderate dose of MDMA administered under conditions that model recreational drug use induces robust, but highly variable increases in brain and body temperatures. These highly variable MDMA-induced temperature responses, ranging from barely detectable increases to pathological hyperthermia, are also typical in humans; the same drug dose could have minimal or no adverse effects in some individuals, but serious life-threatening complications in others (Kalant, 2001; Parrott, 2013a). Our study focused on acute MDMA intoxication, but heightened brain temperature can also cause long-term changes in neural functions, as found in both preclinical studies and human research (Malberg and Seiden, 1998; Parrott and Young, 2014). Variability in temperature responses may explain the large individual differences in the emergence of adverse psychological and cognitive symptoms seen in some longterm MDMA users (for review, see Parrott, 2013b). Therefore, despite the modest hyperthermic responses to MDMA seen in humans under standard laboratory conditions (Freedman et al, 2005; Liechti, 2014), and its potential as a therapeutic agent for post-traumatic stress disorder (Mithoefer et al, 2011, 2013), our study underscores the possible dangers of 'recreational' MDMA use. 
Current emergency therapeutic options to counteract MDMA-induced pathological hyperthermia mainly focus on whole-body cooling, water substitution, and sedative therapy. Indeed, body cooling should have a hypothermic effect, but the effectiveness of this treatment is limited due to strong, sustained MDMA-induced vasoconstriction and the natural vasoconstrictive effect of skin cooling. Similarly, water consumption and/or saline infusions are minimally effective in counteracting MDMA-induced hyperthermia (Proulx et al, 2003). To the best of our knowledge, our study is the first to compare the effectiveness of clozapine and carvedilol with respect to brain hyperthermia induced by MDMA under conditions that mimic human drug use. Using our three-point thermorecording procedure, we were able to clarify the basic physiological mechanisms underlying the therapeutic actions of these drugs.

A clear limitation of our study is the use of a single dose for all therapeutic agents. However, the purpose of this study was not to determine the optimal dosages for each drug, but rather to determine the potential utility of drugs targeting basic physiological mechanisms, specifically, heat production and heat dissipation. Further work will be necessary to establish dose-response relationships for the different drugs to identify the optimal dose range for achieving maximal therapeutic efficacy and minimal side effects.

In conclusion, our data indicate that carvedilol, by acting directly on blood vessels, is modestly effective in attenuating MDMA-induced brain and body hyperthermia. In contrast, clozapine induces much more rapid and powerful hypothermic effects by both decreasing MDMA-induced brain activation and diminishing the sympathetic outflow to peripheral vessels. A therapeutic agent such as clozapine that not only mitigates, but reverses, MDMA-induced hyperthermia could be indispensable for emergency situations and could save the lives of highly intoxicated individuals.

\section{FUNDING AND DISCLOSURE}

The authors declare no conflict of interest.

\section{ACKNOWLEDGMENTS}

We greatly appreciate the great expertize and valuable assistance of Dr Charles Spivak in dissolving drugs used in this study. This study is supported by the National Institute on Drug Abuse-Intramural Research Program, NIH. EAK and YS designed the research; EAK and SR performed the research; EAK and SR analyzed the data; EAK, SR, YS, MHB and KTW wrote the paper. The authors declare no conflict of interest.

\section{REFERENCES}

Bakris G (2009). An in-depth analysis of vasodilation in the management of hypertension: focus on adrenergic blockade. J Cardiovasc Pharmacol 53: 379-387.

Baldessarini RJ, Frankenburg FR (1991). Clozapine. N Engl J Med 324: 746-754.

Baumann MH, Zolkowska D, Kim I, Scheidweiler KB, Rothman RB, Huestis MA (2009). Effects of dose and route of administration on pharmacokinetics of $( \pm)-3,4$-Methylenedioxymethamphetamine in the rat. Drug Metab Dispos 37: 2163-2170.
Blessing W, Seaman B, Pedersen N, Ootsuka Y (2003). Clozapine reverses hyperthermia and sympathetically mediated cutaneous vasoconstriction induced by 3, 4-methylenedioxymethamphetamine (ecstasy) in rabbits and rats. J Neurosci 23: 6385-6391.

Breier A, Buchanan RW, Waltrip Ii RW, Listwak S, Holmes C, Goldstein DS (1994). The effect of clozapine on plasma norepinephrine: relationship to clinical efficacy. Neuropsychopharmacology 10: 1-7.

Brown PL, Kiyatkin EA (2004). Brain hyperthermia induced by MDMA ('ecstasy'): modulation by environmental conditions. Eur J Neurosci 20: 51-58.

Carvedilol (2012). HSDB (Internet). National Library of Medicine: Bethesda, MD, USA. Hazardous Substances Databank Number: 7044. Available from http://toxnet.nlm.nih.gov/cgi-bin/sis/search/ a?dbs+hsdb:@term+@DOCNO+7044.

Charkoudian N (2010). Mechanisms and modifiers of reflex induced cutaneous vasodilation and vasoconstriction in humans. J Appl Physiol 109: 1221-1228.

Dao CK, Nowinski SM, Mills EM (2014). The heat is on: molecular mechanisms of drug-induced hyperthermia. Temperature 1: 183-191.

Dewhirst MW, Viglianti BL, Lora-Michiels $M$, Hanson $M$, Hoopes PJ (2003). Basic principles of thermal dosimetry and thermal thresholds for tissue damage from hyperthermia. Int $J$ Hyperthermia 19: 267-294.

Di Chiara G (2002). Nucleus accumbens shell and core dopamine: differential role in behavior and addiction. Behav Brain Res 137: 75-114.

Freedman RR, Johanson CE, Tancer ME (2005). Thermoregulatory effects of 3,4-methylenedioxymethamphetamine (MDMA) in humans. Psychopharmacology (Berl) 183: 248-256.

Gordon CJ, Watkinson WP, O'Callaghan JP, Miller DB (1991). Effects of 3,4-methylenedioxymethamphetamine on autonomic thermoregulatory responses of the rat. Pharmacol Biochem Behav 38: $339-344$.

Gowing LR, Henry-Edwards SM, Irvine RJ, Ali RL (2002). The health effects of ecstasy: a literature review. Drug Alcohol Rev 21: $53-63$.

Hall AP, Henry JA (2006). Acute toxic effects of 'Ecstasy' (MDMA) and related compounds: overview of pathophysiology and clinical management. Br J Anaesth 96: 678-685.

Hardman HF, Haavik CO, Seevers MH (1973). Relationship of the structure of mescaline and seven analogs to toxicity and behavior in five species of laboratory animals. Toxicol Appl Pharmacol 25: 299-309.

Hysek C, Schmid Y, Rickli A, Simmler L, Donzelli M, Grouzmann E et al (2012). Carvedilol inhibits the cardiostimulant and thermogenic effects of MDMA in humans. $\mathrm{Br} J$ Pharmacol 166: 2277-2288.

Kalant H (2001). The pharmacology and toxicology of 'ecstasy' (MDMA) and related drugs. CMAJ 165: 917-928.

Kellogg DL Jr (2006). In vivo mechanisms of cutaneous vasodilation and vasoconstriction in humans during thermoregulatory challenges. J Appl Physiol 100: 1709-1718.

Kiyatkin EA (2005). Brain hyperthermia as physiological and pathological phenomena. Brain Res Rev 50: 27-56.

Kiyatkin EA (2010). Brain temperature homeostasis: physiological fluctuations and pathological shifts. Front Biosci 15: 73.

Kiyatkin EA, Kim AH, Wakabayashi KT, Baumann MH, Shaham Y (2014). Critical role of peripheral vasoconstriction in fatal brain hyperthermia induced by MDMA (Ecstasy) under conditions that mimic human drug use. J Neurosci 34: 7754-7762.

Liechti ME (2014). Effects of MDMA on body temperature in humans. Temperature 1: 192-200.

Lindt S, Lauener H, Eichenberger E (1971). The toxicology of 8-chloro-11-(4-methyl-1-piperazinyl)-5H-dibenzo(b,e)(1,4)diazepine (clozapine). Farmaco Prat 26: 585-602.

Malberg JE, Seiden LS (1998). Small changes in ambient temperature cause large changes in 3,4-methylenedioxymethamphetamine 
(MDMA)-induced serotonin neurotoxicity and core body temperature in the rat. J Neurosci 18: 5086-5094.

Mechan AO, Esteban B, O'Shea E, Elliott JM, Colado MI, Green AR (2002). The pharmacology of the acute hyperthermic response that follows administration of 3, 4-methylenedioxymethamphetamine (MDMA,'ecstasy') to rats. Br J Pharmacol 135: 170-180.

Mithoefer MC, Wagner MT, Mithoefer AT, Jerome L, Doblin R (2011). The safety and efficacy of $\{+/-\} 3,4-$ methylenedioxymethamphetamine-assisted psychotherapy in subjects with chronic, treatmentresistant posttraumatic stress disorder: the first randomized controlled pilot study. J Psychopharmacol 25: 439-452.

Mithoefer MC, Wagner MT, Mithoefer AT, Jerome L, Martin SF, Yazar-Klosinski B et al (2013). Durability of improvement in post-traumatic stress disorder symptoms and absence of harmful effects or drug dependency after 3,4-methylenedioxymethamphetamine-assisted psychotherapy: a prospective longterm follow-up study. J Psychopharmacol 27: 28-39.

Mogenson GJ, Jones DL, Yim CY (1980). From motivation to action: Functional interface between the limbic system and the motor system. Prog Neurobiol 14: 69-97.

Mueller PD, Korey WS (1998). Death by 'ecstasy': the serotonin syndrome? Ann Emerg Med 32: 377-380.

Parrott AC (2005). Chronic tolerance to recreational MDMA (3,4methylenedioxymethamphetamine) or Ecstasy. J Psychopharmacol 19: 71-83.

Parrott AC (2012). MDMA and temperature: a review of the thermal effects of 'Ecstasy' in humans. Drug Alcohol Depend 121: 1-9.

Parrott AC (2013a). Human psychobiology of MDMA or 'Ecstasy': an overview of 25 years of empirical research. Hum Psychopharmacol 28: 289-307.

Parrott AC (2013b). MDMA, serotonergic neurotoxicity, and the diverse functional deficits of recreational 'Ecstasy' users. Neurosci Biobehav Rev 37: 1466-1484.
Parrott AC, Young L (2014). Saturday night fever: increased core body temperature in dance clubbers on ecstasy/MDMA. Temperature 1: 214-219.

Proulx CI, Ducharme MB, Kenny GP (2003). Effect of water temperature on cooling efficiency during hyperthermia in humans. J Appl Physiol 94: 1317-1323.

Ruffolo RR Jr, Gellai M, Hieble JP, Willette RN, Nichols AJ (1990). The pharmacology of carvedilol. Eur J Clin Pharmacol 38: S82-S88.

Rumana CS, Gopinath SP, Uzura M, Valadka AB, Robertson CS (1998). Brain temperature exceeds systemic temperature in headinjured patients. Crit Care Med 26: 562-567.

Shioda K, Nisijima K, Yoshino T, Kuboshima K, Iwamura T, Yui K et al (2008). Risperidone attenuates and reverses hyperthermia induced by 3,4-methylenedioxymethamphetamine (MDMA) in rats. Neurotoxicology 29: 1030-1036.

Sponer G, Strein K, Bartsch W, Muller-Beckmann B (1992). Vasodilatory action of carvedilol. J Cardiovasc Pharmacol 19: S5-11.

Sprague JE, Moze P, Caden D, Rusyniak DE, Holmes C, Goldstein DS et al (2005). Carvedilol reverses hyperthermia and attenuates rhabdomyolysis induced by 3, 4-methylenedioxymethamphetamine (MDMA, Ecstasy) in an animal model. Crit Care Med 33: 1311-1316.

Taffe MA (2012). Delta9-Tetrahydrocannabinol attenuates MDMAinduced hyperthermia in rhesus monkeys. Neuroscience 201: 125-133.

Tomlinson B, Cronin CJ, Graham BR, Prichard BN (1987). Haemodynamics of carvedilol in normal subjects compared with propranolol, pindolol, and labetalol. J Cardiovasc Pharmacol 10: S69-S75.

Wise RA, Bozarth MA (1987). A psychomotor stimulant theory of addiction. Psychol Rev 94: 469.

Yeh SY (1997). Effects of salicylate on 3,4-methylenedioxymethamphetamine (MDMA)-induced neurotoxicity in rats. Pharmacol Biochem Behav 58: 701-708. 